\title{
Phosphatidylinositol 3,4,5-Trisphosphate 5-Phosphatase 1
}

National Cancer Institute

\section{Source}

National Cancer Institute. Phosphatidylinositol3,4,5-Trisphosphate 5-Phosphatase 1. NCI Thesaurus. Code C95113.

Phosphatidylinositol 3,4,5-trisphosphate 5-phosphatase 1 (1189 aa, 134 kDa) is encoded by the human INPP5D gene. This protein is involved in the removal of phosphate from phosphatidylinositol trisphosphate. 